\title{
Características epidemiológicas dos casos de sífilis congênita no Estado da Paraíba
}

\author{
Epidemiological characteristics of cases of congenital syphilis in the State of Paraíba \\ Características epidemiológicas de los casos de sífilis congénita en el Estado de Paraíba
}

Recebido: 03/03/2021 | Revisado: 18/03/2021 | Aceito: 08/04/2021 | Publicado: 18/04/2021

\author{
Ianne Stéfani Angelim Vieira \\ ORCID: https://orcid.org/0000-0002-9987-8651 \\ Centro Universitário de Patos, Brasil \\ E-mail: iannevieira.enf@gmail.com \\ Mona Lisa Lopes dos Santos Caldas \\ ORCID: https://orcid.org/0000-0002-3701-2836 \\ Centro Universitário de Patos, Brasil \\ E-mail: monalisalopes13@gmail.com \\ Hellen Renatta Leopoldino Medeiros \\ ORCID: https://orcid.org/0000-0001-9444-3774 \\ Centro Universitário de Patos, Brasil \\ E-mail: hellen.medeiros@gmail.com \\ Thoyama Nadja Felix de Alencar Lima \\ ORCID: https://orcid.org/0000-0002-1887-705X \\ Centro Universitário de Patos, Brasil \\ E-mail: thoyamanadja@hotmail.com \\ Eitan Naaman Berezin \\ ORCID: https://orcid.org/0000-0003-4575-0430 \\ Faculdades de Ciências Médicas da Santa Casa de São Paulo, Brasil \\ E-mail: eberezin2003@yahoo.com
}

\begin{abstract}
Resumo
Esse estudo teve como objetivo descrever as características epidemiológicas da sífilis congênita, no estado da Paraíba, durante o período de 2014 a 2019. Trata-se de uma pesquisa do tipo transversal, descritiva com abordagem quantitativa, realizada através do banco de dados do SINAN (Sistema de Informações de Agravos de Notificação), disponibilizados pelo DATASUS (Departamento de informação do Sistema Único de Saúde). Após a aplicação dos critérios de inclusão e exclusão, os dados foram organizados no programa Microsoft EXCEL 2013® para análise das variáveis e disposição das tabelas. A incidência de sífilis congênita foi crescente no estado da Paraíba, a qual aumentou de 4,4 casos para 6,7/1.000 nascidos vivos. Dos 1639 casos notificados, 1593 (97,2\%) possuíam até 6 dias de vida e $1468(89,6 \%)$ foram classificados como sífilis congênita precoce. Relativo às características da mãe, 851 (51,9\%) tinham idade entre 20 e 29 anos, $1377(84,0 \%)$ se autodeclararam pardas, $882(53,8 \%)$ estudaram até o ensino fundamental incompleto ou completo, $1400(85,4 \%)$ realizaram o pré-natal, $791(48,3 \%)$ obtiveram o diagnóstico durante o pré-natal, $1115(68,0 \%)$ foram tratadas inadequadamente e $1010(61,6 \%)$ não tiveram seus parceiros tratados. Assim, verificou-se que a proposta de identificar as características epidemiológicas dos casos notificados de sífilis congênita no estado da Paraíba constitui-se uma ferramenta efetiva para dimensionar a proporção do agravo e para orientar as ações de controle e eliminação da sífilis congênita no estado.
\end{abstract}

Palavras-chave: Sífilis congênita; Características epidemiológicas; Sífilis.

\begin{abstract}
This study aimed to describe the epidemiological characteristics of congenital syphilis, in the state of Paraíba, from 2014 to 2019. This is a cross-sectional, descriptive study with a quantitative approach, carried out through the SINAN database (Information System for Notifiable Diseases), made available by DATASUS (Information Department of the Unified Health System). After applying the inclusion and exclusion criteria, the data were organized in the Microsoft EXCEL $2013{ }^{\circledR}$ program for analyzing the variables and arranging the tables. The incidence of congenital syphilis was increasing in the state of Paraíba, which increased from 4.4 cases to 6.7 / 1,000 live births. Of the 1639 reported cases, $1593(97.2 \%)$ were up to 6 days old and 1468 (89.6\%) were classified as early congenital syphilis. Regarding the characteristics of the mother, 851 (51.9\%) were aged between 20 and 29 years, 1377 (84.0\%) declared themselves brown, $882(53.8 \%)$ studied up to incomplete or complete elementary school, $1400(85.4 \%)$ performed prenatal care, $791(48.3 \%)$ obtained the diagnosis during prenatal care, $1115(68.0 \%)$ were treated inadequately and $1010(61.6 \%)$ did not have their partners treated. Thus, it was found that the proposal to identify the epidemiological characteristics of notified cases of congenital syphilis in the state of Paraíba is an effective tool to measure the proportion of the disease and to guide the actions for the control and elimination of congenital syphilis in the state.
\end{abstract}

Keywords: Congenital syphilis; Epidemiological characteristics; Syphilis. 


\begin{abstract}
Resumen
El presente estudio tuvo como objetivo describir las características epidemiológicas de la sífilis congénita, en el estado de Paraíba, de 2014 a 2019. Se trata de un estudio descriptivo transversal con enfoque cuantitativo, realizado a través de la base de datos SINAN (Sistema de Información de Enfermedades Notificables)., puesto a disposición por DATASUS (Departamento de Información del Sistema Único de Salud). Después de aplicar los criterios de inclusión y exclusión, los datos se organizaron en el programa Microsoft EXCEL 2013® para analizar las variables y ordenar las tablas. La incidencia de sífilis congénita estaba aumentando en el estado de Paraíba, que pasó de 4,4 casos a 6,7 / 1.000 nacidos vivos. De los 1639 casos notificados, $1593(97,2 \%)$ tenían hasta 6 días de vida y $1468(89,6 \%)$ se clasificaron como sífilis congénita temprana. En cuanto a las características de la madre, 851 (51,9\%) tenían entre 20 y 29 años, $1377(84,0 \%)$ se declararon morenas, $882(53,8 \%)$ cursaron hasta primaria incompleta o completa, 1400 $(85,4 \%)$ realizaron prenatales atención, $791(48,3 \%)$ obtuvieron el diagnóstico durante la atención prenatal, 1115 $(68,0 \%)$ fueron tratadas de forma inadecuada y $1010(61,6 \%)$ no recibieron tratamiento a sus parejas. Así, se encontró que la propuesta para identificar las características epidemiológicas de los casos notificados de sífilis congénita en el estado de Paraíba es una herramienta eficaz para medir la proporción de la enfermedad y orientar las acciones para el control y eliminación de la sífilis congénita en el país. Expresar.
\end{abstract}

Palabras clave: Sífilis congénita; Características epidemiológicas; Sífilis.

\title{
1. Introdução
}

Caracterizada como problema de saúde pública, a sífilis congênita está associada principalmente às gestantes infectadas pelo Treponema Pallidum que não foram submetidas aos exames diagnósticos para sífilis, que foram tratadas inadequadamente ou aquelas que não realizaram tratamento algum. Nestes casos, há maior probabilidade dessas gestantes apresentarem desfechos desfavoráveis da gestação, como morte fetal e neonatal, prematuros de baixo peso e crianças infectadas (Padovani, Oliveira \& Pelloso, 2018; Magalhães et al., 2013).

Mundialmente, a Organização Pan-Americana de Saúde (OPAS) e a Organização Mundial de Saúde (OMS) criaram iniciativas para eliminação da infecção mãe-filho até 2015, com objetivo de diminuir a de incidência da sífilis congênita para igual ou menor que 0,5 caso para cada mil nascidos vivos, estabelecendo que, no mínimo $90 \%$ das gestantes, tenham o diagnóstico precoce e o tratamento adequado para infecção (Nunes et al., 2018).

No Brasil, segundo o Boletim Epidemiológico (2018) entre os anos de 2010 a 2017, a taxa de incidência da sífilis cresceu consideravelmente, principalmente no ano de 2016, que apresentou aumento de 31,8\% de sífilis adquirida, 28,5\% em gestantes e 16,4\% da sífilis congênita, podendo está indiretamente ligado à alteração no critério de definição dos casos destes indicadores ocorrida em 2017 (Brasil, 2018).

Em 2011, o Ministério da Saúde (MS) visando atenção humanizada e segura no acompanhamento da gestante e da criança durante o pré-natal, parto e puerpério, foi criado a estratégia "Rede Cegonha" através do Sistema Único de Saúde (SUS). Assegurando, desta forma, a realização da testagem rápida para sífilis no primeiro e terceiro trimestre como também teste sorológico de Venereal Disease Research Laboratory (VDRL) (Moreira et al., 2017).

O Pré-natal é um elemento primordial para redução dos índices de transmissão vertical da sífilis, entretanto, a baixa qualidade deste acompanhamento dificulta o alcance das metas propostas pela OPAS e OMS. Uma vez que mulheres infectadas geralmente iniciam o pré-natal de forma tardia, apresentam baixo número de consultas e não realizam no mínimo um ou dois testes sorológicos, como também registro insuficiente de sorologia reagente no cartão da gestante (Domingues \& Leal, 2016).

Desde o ano de 1986, a sífilis congênita foi incluída na lista de doenças de notificação compulsória no território nacional através da publicação da Portaria $n^{\circ}$ 542/1986, no qual todo caso de recém-nascido, natimorto ou aborto de mulher com sífilis, não submetida ao tratamento ou não tratada adequadamente e toda criança menor de 13 anos apresentando suspeita clínico-epidemiológica da sífilis congênita deve ser devidamente informado ao Sistema de Informação de Agravos de Notificação (Sinan) (Lafetá et al., 2016; Brasil, 2017).

Apesar da existência de equipes de saúde da família, há quem fique sem acesso aos serviços de saúde e 
consequentemente sem diagnóstico e tratamento precoce, firmando a falta da qualidade na atenção e as falhas nos tratamentos das grávidas infectadas e seus parceiros, que são pontos vulneráveis da assistência obstétrica e neonatal, motivando um desafio para as ações de controle da sífilis (Flores, 2011).

Neste cenário, o conhecimento do contexto epidemiológico local da sífilis congênita sugere a questão a ser investigada: Quais as características epidemiológicas dos casos de sífilis congênita no estado da Paraíba?

Sendo assim, a escolha do tema justifica-se pela necessidade de conhecer o perfil epidemiológico acerca do assunto no estado da Paraíba, pois percebe-se a relevância da sífilis congênita no âmbito da saúde pública e a importância da avaliação do perfil de toda e qualquer infecção sexualmente transmissível (IST) que possa trazer prejuízos para a gestante ou para o bebê, visando conhecer a realidade de cada região e/ou estado.

Tendo em vista o exposto acima, este estudo teve por objetivo identificar as características epidemiológicas dos casos de sífilis congênita no estado da Paraíba no período de 2014 a 2019.

\section{Metodologia}

Trata-se de uma pesquisa epidemiológica, ecológica e descritiva, de série temporal com abordagem quantitativa. Os estudos ecológicos avaliam os contextos sociais e ambientais que afetam a saúde de grupos populacionais, abrangendo, assim, os estudos epidemiológicos (Bezerra Filho et al., 2012). Relativo aos métodos quantitativos, estes permitem gerar conjuntos ou massas de dados que podem ser analisados por meio de técnicas matemáticas como é o caso das porcentagens, possibilitando a previsão de acontecimentos (Pereira et al., 2018).

A pesquisa foi realizada através do banco de dados do SINAN (Sistema de Informações de Agravos de Notificação) disponibilizados pelo Departamento de Informática do Sistema Único de Saúde (DATASUS), no período de janeiro de 2014 a dezembro de 2019.

O presente estudo foi composto por todos os nascidos vivos, no período de 2014 a 2019, e a amostra constituída por todos os casos confirmados de sífilis congênita registrados no SINAN no período de escolha. O instrumento de coleta usado foram os dados contidos na ficha de notificação compulsória de sífilis congênita disponibilizados pelo DATASUS.

O procedimento para coleta dos dados originou-se a partir do acesso ao banco de dados do SINAN, no qual foram levantados os casos de sífilis congênita do estado da Paraíba que foram notificados pelos estabelecimentos de saúde do estado ao DATASUS, no recorte histórico formado pelos anos de 2014 a 2019. Após esta etapa, os dados foram transformados e exportados por meio de tabela do software do Microsoft EXCEL 2013®. As variáveis analisadas consistiram em: faixa etária, faixa etária da mãe, raça da mãe, escolaridade da mãe, realização do pré-natal, momento do diagnóstico da sífilis materna, esquema de tratamento da mãe, tratamento do parceiro, diagnóstico final e óbitos da doença.

Como critérios de inclusão foram inseridos todos os casos confirmados de sífilis congênita do estado da Paraíba, disponíveis no Sistema de Informações de Agravos de Notificação - SINAN e excluídos os casos notificados registrados fora do período de estudo.

Os dados foram coletados e analisados durante os meses de agosto a novembro de 2020 e inicialmente tabulados através do programa TABNET do DATASUS, gerando o banco de dados com as variáveis usadas nesta pesquisa. Posteriormente, utilizou-se o programa Microsoft EXCEL 2013® para análise das variáveis e disposição das tabelas. Os resultados foram apresentados em números absoluto e em percentual (\%). Foi calculada a taxa de incidência por 1.000 nascidos vivos e dos óbitos o coeficiente bruto de mortalidade por 100.000 nascidos vivos.

\section{Resultados}

Verificou-se que dos 290.405 nascidos vivos entre 2014 e 2018 na Paraíba, 1.639 crianças foram diagnosticadas com 
a doença, o que determina uma taxa anual média de incidência de 5,0. O ano com maior número de casos foi o de 2017, com 395 notificações entre 57.493 nascidos vivos, resultando em uma taxa de incidência por 1.000 nascidos vivos de 6,8 (Tabela $1)$.

De acordo com os dados, a Paraíba apresentou, em 2016, uma diminuição do número de casos, comparado aos outros anos analisados. Segundo o Boletim Epidemiológico (2018), esta diminuição ocorreu devido a erros na informação dos dados do nível estadual para o nível nacional. Podendo o aumento do ano de 2017 fazer referência à reparação do número de notificações não informadas no ano anterior e não um aumento real de casos de sífilis congênita.

Tabela 1. Distribuição das variáveis sociais e epidemiológicas dos casos de sífilis congênita na Paraíba, no período entre 20142019. Apresentação dos resultados: $\mathrm{n}^{\circ}$ de casos (\% percentual).

\begin{tabular}{lccccccc}
\hline & $\mathbf{2 0 1 4}$ & $\mathbf{2 0 1 5}$ & $\mathbf{2 0 1 6}$ & $\mathbf{2 0 1 7}$ & $\mathbf{2 0 1 8}$ & $\mathbf{2 0 1 9}$ & Total \\
Casos confirmados & $252(15,4)$ & $319(19,5)$ & $85(5,2)$ & $395(24,1)$ & $383(23,4)$ & $205(12,5)$ & 1.639 \\
Incidência & 4,4 & 5,4 & 1,5 & 6,8 & 6,7 & - & 5,0 \\
Idade & & & & & & & \\
Até 6 dias & $248(98,4)$ & $305(95,6)$ & $79(92,9)$ & $388(98,2)$ & $372(97,1)$ & $201(98,0)$ & $1.593(97,2)$ \\
7-27 dias & $1(0,4)$ & $8(2,5)$ & $4(4,7)$ & $4(1,0)$ & $6(1,6)$ & $1(0,5)$ & $24(1,5)$ \\
$\mathbf{2 8 ~ d i a s ~ a ~ < 1 ~ a n o ~}$ & $3(1,2)$ & $5(1,6)$ & $2(2,4)$ & $1(0,3)$ & $5(1,3)$ & $3(1,5)$ & $19(1,2)$ \\
$\mathbf{1}$ ano (12 a 23 meses) & 0 & $1(0,3)$ & 0 & $1(0,3)$ & 0 & 0 & $2(0,1)$ \\
$\mathbf{2}$ a 4 anos & 0 & 0 & 0 & $1(0,3)$ & 0 & 0 & $1(0,1)$ \\
$\mathbf{5}$ a 12 anos & 0 & 0 & 0 & 0 & 0 & 0 & 0 \\
Diagnóstico final & & & & & & & \\
Sífilis congênita recente & $219(86,9)$ & $279(87,5)$ & $81(95,3)$ & $355(89,9)$ & $345(90,1)$ & $189(92,2)$ & $1468(89,6)$ \\
Sífilis congênita tardia & 0 & 0 & 0 & $1(0,3)$ & 0 & 0 & $1(0,1)$ \\
Aborto por sífilis & $14(5,6)$ & $19(6,0)$ & $1(1,2)$ & $14(3,5)$ & $23(6,0)$ & $7(3,4)$ & $78(4,8)$ \\
Natimorto por sífilis & $19(7,5)$ & $21(6,6)$ & $3(3,5)$ & $25(6,3)$ & $15(3,9)$ & $9(4,4)$ & $92(5,6)$ \\
Óbitos & & & & & & & \\
Casos & $1(0,4)$ & $1(0,3 \%)$ & $4(4,7)$ & $1(0,3 \%)$ & $4(1,0)$ & - & $11(0,7)$ \\
Coeficiente & 1,7 & 1,7 & 7,1 & 1,7 & 7,0 & - & - \\
\hline
\end{tabular}

Incidência: casos/1.000 nascidos vivos. Coeficiente: casos/100.000 nascidos vivos.

Fonte: organização da autora com dados procedentes do SINAN (2014-2019).

Com relação à notificação de acordo com a faixa etária dos recém-nascidos, observou-se uma maior predominância nos RN de até 6 dias, que se manteve ao longo dos anos, com 1.593 (97,2\%) casos de 2014 a 2019, sendo o ano de 2017 com o maior número de casos (388). Cabe ressaltar que 99,9\% dos RN foram diagnosticados até o primeiro ano de vida.

Do diagnóstico final dos casos notificados, 1.468 (89,6\%) foram classificados como sífilis congênita recente, 92 $(5,6 \%)$ natimorto por sífilis, 78 (4,8\%) como aborto por sífilis e $1(0,1 \%)$ caso de sífilis congênita tardia.

Relativo aos óbitos por sífilis congênita, como pode ser observado, dos 1639 casos notificados como sífilis congênita, no período analisado, foram a óbito 11 (0,7\%) crianças. Nos anos de 2014, 2015 e 2017, ocorreu apenas 1 óbito, o que representou um coeficiente de mortalidade de 1,7/100.000 nascidos vivos. Entretanto, nos anos de 2016 e 2018 houve um aumento e 4 crianças morreram devido à doença, perfazendo um coeficiente de 7,1\% e 7,0\%, respectivamente, nestes anos.

No que diz respeito às características da mãe, nesta pesquisa predominaram as mães com faixa etária entre 20 a 29 anos $(51,9)$, seguidas daquelas com idade de 15 a 19 anos $(25,5 \%)$ e 30 a 39 anos $(17,9 \%)$. 
Tabela 2. Distribuição das variáveis sociodemográficas, obstétrica e epidemiológicas das genitoras dos casos de sífilis congênita na Paraíba, no período entre 2014-2019. Apresentação dos resultados: $n^{\circ}$ de casos (\% percentual).

\begin{tabular}{|c|c|c|c|c|c|c|c|}
\hline Idade & 2014 & 2015 & 2016 & 2017 & 2018 & 2019 & Total \\
\hline 10 a 14 anos & $2(0,8)$ & $5(1,6)$ & $2(2,4)$ & $3(0,8)$ & $5(1,3)$ & $2(1,0)$ & $19(1,2)$ \\
\hline 15 a 19 anos & $65(25,8)$ & $78(24,5)$ & $19(22,4)$ & $98(24,8)$ & $102(26,6)$ & $56(27,3)$ & $418(25,5)$ \\
\hline 20 a 29 anos & $139(55,2)$ & $142(44,5)$ & $47(55,3)$ & $209(52,9)$ & $204(53,3)$ & $110(53,7)$ & $851(51,9)$ \\
\hline 30 a 39 anos & $43(17,1)$ & $63(19,7)$ & $17(20,0)$ & $76(19,2)$ & $62(16,2)$ & $32(15,6)$ & $293(17,9)$ \\
\hline 40 anos ou mais & $2(0,8)$ & $2(0,6)$ & 0 & $4(1,0)$ & $6(1,6)$ & $4(2,0)$ & $18(1,1)$ \\
\hline Ignorado & $1(0,4)$ & $29(9,1)$ & 0 & $5(1,3)$ & $4(1,0)$ & $1(0,5)$ & $40(2,4)$ \\
\hline \multicolumn{8}{|l|}{ Raça } \\
\hline Branca & $16(6,3)$ & $20(6,3)$ & $2(2,4)$ & $47(11,9)$ & $41(10,7)$ & $22(10,7)$ & $148(9,0)$ \\
\hline Preta & $13(5,2)$ & $16(5,0)$ & $3(3,5)$ & $11(2,8)$ & $10(2,6)$ & $8(3,9)$ & $61(3,7)$ \\
\hline Amarela & 0 & 0 & 0 & $1(0,3)$ & 0 & 0 & $1(0,1)$ \\
\hline Parda & $213(84,5)$ & $262(82,1)$ & $73(85,9)$ & $323(81,8)$ & $331(86,4)$ & $175(85,4)$ & $1377(84,0)$ \\
\hline Indígena & $2(0,8)$ & $1(0,3)$ & 0 & 0 & 0 & 0 & $3(0,2)$ \\
\hline Ignorada & $8(3,2)$ & $20(6,3)$ & $7(8,2)$ & $13(3,3)$ & $1(0,3)$ & 0 & $49(3,0)$ \\
\hline \multicolumn{8}{|l|}{ Escolaridade } \\
\hline Analfabeto & $7(2,8)$ & $7(2,2)$ & 0 & $2(0,5)$ & $6(1,6)$ & $1(0,5)$ & $23(1,4)$ \\
\hline $1^{\mathrm{a}}$ a $4^{\mathrm{a}}$ série incomp. & $31(12,3)$ & $27(8,5)$ & $5(5,9)$ & $24(6,1)$ & $27(7,0)$ & $9(4,4)$ & $123(7,5)$ \\
\hline $4^{\mathrm{a}}$ série completa & $13(5,2)$ & $21(6,6)$ & $2(2,4)$ & $9(2,3)$ & $23(6,0)$ & $21(10,2)$ & $89(5,4)$ \\
\hline $5^{\mathrm{a}}$ a $8^{\mathrm{a}}$ série incomp. & $85(33,7)$ & $90(28,2)$ & $19(22,4)$ & $92(23,3)$ & $103(26,9)$ & $60(29,3)$ & $449(27,4)$ \\
\hline Fundamental completo & $17(6,7)$ & $35(11,0)$ & $21(24,7)$ & $52(13,2)$ & $42(11,0)$ & $31(15,1)$ & $198(12,1)$ \\
\hline Médio incomp. & $11(4,4)$ & $16(5,0)$ & $4(4,7)$ & $23(5,8)$ & $26(6,8)$ & $13(6,3)$ & $93(5,7)$ \\
\hline Médio completo & $35(13,9)$ & $44(13,8)$ & $8(9,4)$ & $40(10,1)$ & $57(14,9)$ & $26(12,7)$ & $210(12,8)$ \\
\hline Superior incomp. & $1(0,4)$ & $2(0,6)$ & 0 & $3(0,8)$ & $2(0,5)$ & $3(1,5)$ & $11(0,7)$ \\
\hline Superior completo & 0 & 0 & $1(1,2)$ & $1(0,3)$ & $3(0,8)$ & $1(0,5)$ & $6(0,4)$ \\
\hline Não se aplica & $2(0,8)$ & $2(0,6)$ & 0 & 0 & $1(0,3)$ & $1(0,5)$ & $6(0,4)$ \\
\hline Ignorado & $50(19,8)$ & $75(23,5)$ & $25(29,4)$ & $149(37,7)$ & $93(24,3)$ & $39(19,0)$ & $431(26,3)$ \\
\hline \multicolumn{8}{|l|}{ Pré-natal } \\
\hline Sim & $211(83,7)$ & $260(81,5)$ & $76(89,4)$ & $339(85,8)$ & $330(86,2)$ & $184(89,8)$ & $1400(85,4)$ \\
\hline Não & $39(15,5)$ & $53(16,6)$ & $6(7,1)$ & $50(12,7)$ & $50(13,1)$ & $19(9,3)$ & $217(13,2)$ \\
\hline Ignorado & $2(0,8)$ & $6(1,9)$ & $3(3,5)$ & $6(1,5)$ & $6(0,8)$ & $2(1,0)$ & $22(1,3)$ \\
\hline \multicolumn{8}{|c|}{ Diagnóstico de sífilis materna } \\
\hline $\begin{array}{l}\text { Durante o pré-natal } \\
\text { No momento do }\end{array}$ & $105(41,7)$ & $136(42,6)$ & $51(60,0)$ & $205(51,9)$ & $180(47,0)$ & $114(55,6)$ & $791(48,3)$ \\
\hline parto/curetagem & $119(47,2)$ & $153(48,0)$ & $24(28,2)$ & $157(39,7)$ & $185(48,3)$ & $82(40,0)$ & $720(43,9)$ \\
\hline Após o parto & $20(7,9)$ & $19(6,0)$ & $8(9,4)$ & $16(4,1)$ & $11(2,9)$ & $5(2,4)$ & $79(4,8)$ \\
\hline Não realizado & $2(0,8)$ & $2(0,6)$ & 0 & $3(0,8)$ & 0 & 0 & $7(0,4)$ \\
\hline Ignorado & $6(2,4)$ & $9(2,8)$ & $2(2,4)$ & $14(3,5)$ & $7(1,8)$ & $4(2,0)$ & $42(2,6)$ \\
\hline \multicolumn{8}{|l|}{ Esquema de tratamento } \\
\hline Adequado & $4(1,6)$ & $5(1,6)$ & $11(12,9)$ & $20(5,1)$ & $24(6,3)$ & $12(5,9)$ & $76(4,6)$ \\
\hline Inadequado & $198(78,6)$ & $229(71,8)$ & $44(51,8)$ & $262(66,3)$ & $244(63,7)$ & $138(67,3)$ & $1115(68,0)$ \\
\hline Não realizado & $30(11,9)$ & $35(11,0)$ & $12(14,1)$ & $65(16,5)$ & $89(23,2)$ & $37(18,0)$ & $268(16,4)$ \\
\hline Ignorado & $20(7,9)$ & $50(15,7)$ & $18(21,2)$ & $48(12,2)$ & $26(6,8)$ & $18(8,8)$ & $180(11,0)$ \\
\hline
\end{tabular}




\begin{tabular}{lccccccc}
\hline Tratamento do parceiro & & & & & & & \\
Sim & $35(13,9)$ & $44(13,8)$ & $21(24,7)$ & $103(26,1)$ & $115(30,0)$ & $78(38,0)$ & $396(24,2)$ \\
Não & $194(77,0)$ & $225(70,5)$ & $41(48,2)$ & $233(59,0)$ & $219(57,2)$ & $98(47,8)$ & $1010(61,6)$ \\
Ignorado & $23(9,1)$ & $50(15,7)$ & $23(27,1)$ & $59(14,9)$ & $49(12,8)$ & $29(14,1)$ & $233(14,2)$ \\
\hline
\end{tabular}

Fonte: organização da autora com dados procedentes do SINAN (2014-2019).

A raça parda $(84,0 \%)$, com 1.377 casos, foi predominante nas genitoras entre 2014 e 2019, seguindo-se as de raça branca $(9,0 \%)$ e as de raça preta $(3,7 \%)$ com 148 e 61 casos respectivamente. Havendo $3(0,2 \%)$ casos de mulheres consideradas indígenas e $1(0,1 \%)$ mulher de raça amarela.

Observou-se que a maior parte das genitoras das crianças notificadas apresentavam baixo nível de escolaridade, no qual $882(53,8 \%)$ mulheres possuíam escolaridade até Ensino Fundamental incompleto ou completo. 303 (18,5\%) apresentaram Ensino Médio incompleto ou completo. Apenas 17 (1,1\%) do total dos casos notificados durante os anos de 2014 a 2019 as genitoras possuíam Ensino Superior, sendo concluído ou não. Em 437 (26,7\%) dos casos foram ignorados ou considerados como "não se aplica" no momento da notificação.

Dos 1639 casos notificados, $1400(85,4 \%)$ mulheres realizaram o pré-natal, existindo 217 (13,2\%) mães que não realizaram e $22(1,3 \%)$ que foram classificadas como sendo branco/ignorado. Constatou-se que durante todos os anos estudados entre 2014 e 2019, a assistência de pré-natal obteve cobertura de mais de $80 \%$, no qual o último ano apresentou o maior percentual de cobertura com $89,8 \%$.

Durante o período de estudo, 791 (48,3\%) mulheres foram informadas do diagnóstico de sífilis materna ainda durante a realização do pré-natal, 720 (43,9\%) apenas no momento do parto/curetagem e $79(4,8)$ após o parto. O diagnóstico não foi obtido por $7(0,4 \%)$ genitoras em nenhum momento da gestação/parto/puerpério, além de 42 (2,6\%) casos com informações ignoradas.

Em relação ao esquema de tratamento da mãe, este foi considerado inadequado em 1.115 (68,0\%) mulheres, não foram realizados em $268(16,4)$ genitoras e ignorado em $180(11,0 \%)$ delas. Apenas $76(4,6 \%)$ mulheres foram tratadas adequadamente para sífilis na gestação.

De um total de 1.639 casos, entre os anos de 2014 e 2019, apenas 396 (24,2\%) parceiros foram tratados e 1.010 não foram tratados (61,6\%). Os outros 233 (14,2\%) foram marcados "ignorado" no momento do preenchimento da ficha.

Observa-se que o número de parceiros tratados vem crescendo ao longo dos últimos anos, o qual passou de 13,9\% em 2014 para 38\% em 2019. Porém, o número de notificações marcadas como "ignorado" para o tratamento do parceiro ainda persiste, o que dificulta a observância do real número de parceiros tratados.

\section{Discussão}

A partir da exposição dos resultados, pode-se identificar que, no estado da Paraíba, a taxa de incidência da sífilis congênita ainda persiste muito alta, demonstrando a necessidade de estratégias que visem o seu controle. Foi possível também verificar que a sífilis congênita acomete principalmente crianças nascidas de mães jovens, de raça/cor parda, com baixo nível de escolaridade, que realizaram o pré-natal, não foram tratadas adequadamente e não tiveram seus parceiros tratados simultaneamente.

Verificou-se na Paraíba, entre os anos de 2014 a 2018, uma taxa de incidência anual média de 5,0 dos casos de sífilis congênita por mil nascidos vivos, ultrapassando 10 vezes o que é preconizado pela OPAS e OMS, que visa diminuir a incidência da sífilis congênita para igual ou menor que 0,5 caso/1.000 nascidos vivos (Nunes et al., 2018).

Segundo Cavalcante et. al. (2017) em estudo desenvolvido na cidade de Palmas capital do estado do Tocantins, de 
2007 a 2014 a taxa de incidência anual de sífilis congênita elevou-se de 2,9 para 8,1 casos por 1.000 nascidos vivos. O autor relata também alguns obstáculos inerentes às pesquisas com dados secundários - condicionados à cobertura e qualidade dos registros -, dada a probabilidade de sub-registros, subnotificações e baixa qualidade das informações registradas.

A ausência de matéria-prima para produção de penicilina foi outro fator contribuinte para o crescimento no número de casos de sífilis congênita, no qual o Ministério da Saúde (MS) publicou em 2015 uma nota informativa orientando a priorização do uso da penicilina $\mathrm{G}$ benzatina na terapêutica da sífilis em gestantes e penicilina cristalina no tratamento da sífilis congênita no país. Já para a sífilis adquirida e em parceiros de gestantes, preconizou-se o uso de outros antibióticos disponíveis no SUS com posologia para tratamento de 8 a 15 dias, contribuindo assim para ocorrência de falhas na sua adesão e consequente reinfecção (Rodrigues, Oliveira \& Afonso, 2017).

$\mathrm{O}$ perfil das genitoras encontrado nesta pesquisa foi semelhante ao descrito em outros estudos. Relativo à taxa de detecção da sífilis congênita associada à faixa etária da mãe, estudos como Farias (2017), Holanda et al. (2011) e Oliveira e Figueiredo (2011) também constataram que a maioria dos casos eram de conceptos de mães jovens entre 20 e 29 anos de idade. A maioria das mulheres que apresentaram desfecho de bebês com sífilis congênita se autodeclararam pardas, o que também foi evidenciado no estudo de Saraceni et al. (2017) sobre sífilis em gestante e sífilis congênita, no qual constatou que das 6 unidades federativas estudadas, 5 estados também apresentaram a cor parda como predominante. Entretanto, apenas o estado do Rio Grande do Sul apresentou $61,4 \%$ de mulheres consideradas de raça branca.

Níveis de escolaridade parecidos foram descritos por Domingues e Leal (2016) em pesquisa nacional de base hospitalar composta por puérperas e seus recém-nascidos nos anos de 2011 e 2012, a qual encontrou maior percentual de mulheres com Ensino Fundamental incompleto em mães de RN com desfecho de sífilis congênita. Observou-se que quanto menor a escolaridade da mulher, maior a ocorrência de infecção pela sífilis e de sífilis congênita.

A gravidez em idade jovem e a baixa escolaridade, portanto, amplia a possibilidade de exposição a infecções sexualmente transmissíveis, visto que o conhecimento a respeito da importância das medidas de precaução é mínimo ou ausente.

Em relação à taxa de realização do pré-natal, estudos semelhantes como de Reis et. al. (2018), realizado no estado de Minas Gerias, entre os anos de 2007 a 2013, detectou que 78,8\% das mães realizaram o pré-natal. O mesmo afere o estudo de Melo (2019), no qual verificou que 78,5\% mulheres analisadas tiveram a mesma assistência. Teixeira et al. (2018) confirma, em sua pesquisa no estado do Rio Grande do Sul (2001-2012), que 77,4\% declararam que também realizaram o pré-natal.

Apesar dos resultados demonstrarem que a cobertura da assistência de pré-natal comportou mais de $80 \%$ do número de genitoras de crianças com sífilis congênita, sabe-se que altas taxas de realização de pré-natal não essencialmente significam qualidade e adequação da assistência. Pois as razões que classificam um pré-natal adequado são atreladas a diversos fatores como idade gestacional no início do pré-natal, número de consultas, realização dos exames de rotina e outros (Padovani; Oliveira; Pelloso, 2018).

Pesquisas realizadas em outros contextos sugerem falhas no manejo dos casos de sífilis na gestação, com predomínio daqueles de sífilis congênita considerados evitáveis. A persistência de alta incidência da doença e de altas taxas de transmissão vertical, mesmo após a ampliação da cobertura do pré-natal e do número médio de consultas com a instalação do Sistema Único de Saúde (SUS), mostra que a qualidade da assistência é insatisfatória (Domingues et al., 2013).

O diagnóstico precoce da sífilis materna ainda é a melhor maneira de prevenir o desfecho de sífilis congênita na gestação, entretanto, constatou-se, neste estudo, que apenas 48,3\% das mulheres receberam o diagnóstico ainda na gestação. Esses resultados corroboram os encontrados por Moreira et al. (2017), o qual descreve nas características maternas dos casos notificados de sífilis congênita que $60,1 \%$ das mães tiveram o diagnóstico de sífilis durante o pré-natal, 28,28\% no momento do parto/curetagem e $9,6 \%$ após o parto. 
Já no estudo de Lafetá et al. (2016), 62,4\% das mulheres só receberam o diagnóstico de sífilis materna após o parto/curetagem, e aquelas que receberam o diagnóstico ainda na gestação $10,7 \%$ estavam no $1^{\circ}$ trimestre e $22,6 \%$ no $2^{\circ} / 3^{\circ}$ trimestre. O mesmo é apresentado por Holanda et al. (2011), o qual aponta que 42,8\% não tiveram o diagnóstico de sífilis durante a gravidez e somente $34,7 \%$ receberam diagnóstico durante a gestação, com baixo percentual de realização do teste VDRL no primeiro e segundo períodos da gestação, o que contraria as recomendações do MS.

Salienta-se que o "tratamento adequado", por definição adotada pelo Ministério da Saúde, é todo tratamento completo com penicilina e adequado para a fase clínica da doença, realizado pelo menos 30 (trinta) dias anteriores ao parto e com o parceiro tratado simultaneamente com a gestante (Farias, 2017). Tendo em vista estes requisitos, é possível que números tão baixos de mães tratadas adequadamente encontrado neste estudo possa estar atribuído ao fato de que mesmo aquelas que receberam tratamento não cumpriam com todos os critérios preconizados.

Elevadas taxas de mães classificadas como tratadas inadequadamente ou que não receberam tratamento algum também é demonstrado no estudo de Cavalcante et al. (2017), no qual a maior parte das genitoras de casos de sífilis congênita notificados não receberam tratamento $(54,4 \%)$, e parte importante recebeu tratamento inadequado $(40,7 \%)$ e a realização da terapêutica adequada da gestante, ao longo dos anos, manteve-se baixa (1,0\%). Holanda et al. (2011) corrobora para esses dados, já que em sua pesquisa foi verificado que $73,0 \%$ das gestantes receberam tratamento não adequado, $6,1 \%$ não se trataram e apenas $4,5 \%$ foram tratadas de forma adequada.

Mesmo a sífilis sendo uma doença conhecida há vários anos e que tenha agente etiológico bem definido, tratamento eficaz e de baixo custo, estabelecido desde o ano de 1943, é ampla a quantidade de gestantes infectadas que não são sujeitas às ações terapêuticas preconizadas pelo o Programa de Humanização do Pré-Natal e Nascimento em relação ao controle e prevenção da transmissão vertical (Domingues et al., 2013).

Neste estudo observou-se que o número de parceiros não tratados vem diminuindo ao longo dos últimos anos, os quais passaram de 77,0\% em 2014 para 47,8\% em 2019. Porém, o número de notificações marcadas como "ignorado" para o tratamento do parceiro ainda persiste, o que dificulta a observância do real número de parceiros realmente tratados. Esses resultados corroboram os encontrados por Lima et al. (2013), em estudo realizado em Belo Horizonte/MG, o qual constatou um percentual de $89 \%$ de parceiros não tratados. Holanda et al. (2011), em estudo realizado no Município de Natal/RN, também verificou índice elevado de não tratados (54\%), com um percentual de 34,7\% de ignorados.

Esses dados demonstram a necessidade de ampliação das ações de controle da sífilis e consequentemente o aumento dos números de tratamento dos parceiros, objetivando evitar a reinfecção da gestante e desfechos desfavoráveis por estas, sendo a falta de realização deste tratamento, ou a realização de tratamento inadequado, um dos critérios estabelecidos pelo Ministério da Saúde para a definição de caso de sífilis congênita.

Segundo o diagnóstico final, a maior parte dos casos foram classificados como sífilis recente, ou seja, estava no primeiro estágio clínico da doença. Nacionalmente, segundo o Boletim Epidemiológico (2018), quanto à classificação final dos casos, verificou-se que 93,2\% foram classificados como sífilis congênita recente, 3,5\% como caso de aborto por sífilis, 3,1\% como natimorto e $0,2 \%$ como sífilis congênita tardia. Correspondentemente, Farias (2017) também evidenciou que segundo os critérios de estadiamento dos casos, $88 \%$ (3.651) das crianças enquadravam-se na classificação de sífilis congênita recente, $6,36 \%$ (264) natimorto e 5,42\% (225) abortos por sífilis.

É importante evidenciar que a sífilis se classifica em dois estágios: sífilis congênita precoce, quando é diagnosticada até dois anos de vida e sífilis congênita tardia, quando diagnosticada após os primeiros dois anos. No entanto, o diagnóstico na criança configura um processo complexo devido à maioria das crianças não apresentarem sintomas ao nascimento e, naquelas com demonstração clínica, os sinais podem ser discretos ou pouco específicos e não existe uma avaliação complementar para definir com exatidão o diagnóstico da infecção na criança. Nesse sentido, salienta-se a associação de critérios epidemiológicos, 
clínicos e laboratoriais para a classificação da sífilis (Brasil, 2005).

Referente aos óbitos por sífilis congênita, como pode ser observado, foram a óbito $11(0,7 \%)$ crianças. Dados parecidos com a pesquisa atual pode ser observado no estudo de Padovani, Oliveira e Pelloso (2018) realizado na $15^{\text {a }}$ Regional de Saúde do Estado do Paraná no período de 2011 a 2015, onde 1,36\% dos recém-nascidos apresentaram óbitos por sífilis congênita. Números ainda maiores foram encontrados por Farias (2017), trazendo em seu estudo que dos 4.149 casos notificados como sífilis congênita, no período de 2010 a 2014, vieram a óbito em decorrência da doença 625 (15,05\%) crianças.

Nesse ínterim, é preciso ressaltar que este estudo constatou que a proposta de identificar as características epidemiológicas dos casos notificados de sífilis congênita no estado da Paraíba constitui-se uma ferramenta efetiva para dimensionar a proporção do agravo e para orientar as ações de controle e eliminação da sífilis congênita no estado. Dessa forma, torna-se de suma importância a propagação desses resultados, de modo que possa subsidiar estratégias diferenciadas que visem à minimização ou eliminação da sífilis como um problema de saúde pública. É preciso considerar as limitações inerentes à característica metodológica da pesquisa como a utilização de dados secundários e a qualidade do preenchimento das fichas de notificação compulsória, que dificultam a precisão dos dados coletados.

\section{Conclusão}

Os resultados desta pesquisa indicam que elevadas taxas de incidência da sífilis congênita no estado da Paraíba e altas taxas de transmissão vertical estão associadas principalmente ao tratamento inadequado das gestantes e parceiros, como também as características sociodemográficas das genitoras, que em sua maioria são jovens, de raça/cor parda e com baixa escolaridade.

De acordo com a análise dos dados obstétricos da mãe, apesar da realização da assistência de pré-natal, muitas gestantes só foram diagnosticadas com sífilis materna no parto/curetagem ou pós-parto, além disso, elas não receberam o tratamento adequado e não tiveram os parceiros tratados concomitantemente, conforme preconizado pelo Ministério da Saúde, reforçando a precariedade da qualidade da assistência.

Portanto, é necessário que as equipes de saúde da atenção primária sejam capacitadas para realização dos atendimentos de pré-natal com qualidade, que inclui a busca da gestante para início precoce do pré-natal, solicitação de exames e obtenção de resultados em tempo oportuno, realização do pré-natal do parceiro visando principalmente tratar adequadamente os casos e realizar orientações sobre a doença e a importância do diagnóstico e tratamento precoce.

Destaca-se neste estudo que há, em muitos casos, o preenchimento incompleto ou em branco dos casos de sífilis congênita no estado da Paraíba, o que infere uma fragilidade dos profissionais sobre a vigilância epidemiológica da doença. Apesar da ficha de notificação ter em anexo um manual de preenchimento, é provável que a leitura do material esteja dificultando o exato preenchimento da ficha de notificação. O preenchimento de forma completa é essencial para o monitoramento da sífilis congênita, além da avaliação dos programas e políticas públicas. Deste modo, é nítida a necessidade de treinamento dos profissionais para este fim.

Para os estudos a serem desenvolvidos a partir deste, sugere-se uma avaliação qualitativa da assistência dos serviços oferecidos no pré-natal, inerentes a identificação da sífilis materna, dificuldades para o tratamento adequado e adesão do parceiro ao pré-natal, com o objetivo de melhor elucidar os principais entraves na eliminação e controle da doença na atenção primária. 


\section{Referências}

Brasil, (2018). Boletim epidemiológico: Sífilis 2018. Secretaria de vigilância em saúde - Ministério da Saúde. 49(45).

Brasil, (2017). Nota informativa n 02-SEI/2017 - DIAHV/SVS/MS. Ministério da Saúde. Brasília-DF.

Brasil, (2005). Diretrizes para o controle da sífilis congênita. Ministério da Saúde - Secretaria de Vigilância em Saúde - Programa Nacional de DST e Aids. Brasília-DF.

Bezerra Filho, J. G., Werneck, G. L., Almeida, R. L. F., Oliveira, M. I. V. \& Magalhães, F. B. (2012). Estudo ecológico sobre os possíveis determinantes socioeconômicos, demográficos e fisiográficos do suicídio no estado do Rio de Janeiro, Brasil 1998-2002. Cad. Saúde Pública, 28(5), 833-844.

Cavalcante, P. A. M., Pereira, R. B. L. \& Castro, J. G. D. (2017). Syphilis in pregnancy and congenital syphilis in Palmas, Tocantins State, Brazil, $2007-2014$. Epidemiologia e Serviços de Saúde [online]. 26(2), 255-264.

Domingues, R. M. S. M. \& Leal, M. C. (2016). Incidência de sífilis congênita e fatores associados à transmissão vertical da sífilis: dados do estudo Nascer no Brasil. Cad. Saúde Pública, 32(6)

Domingues, R. M. S. M., Saraceni, V., Hartz, Z. M. A. \& Leal, M. C. (2013). Sífilis congênita: evento sentinela da qualidade da assistência pré-natal. Revista Saúde Pública47(1), 147-57.

Farias, N. L. L. (2017). Sífilis congênita em Pernambuco: Influência dos fatores sociodemográficos e assistenciais. 40 f. TCC (Residência Multiprofissional em Saúde Coletiva) - Instituto Aggeu Magalhães, Recife.

Flores, R. L. R. (2011). Sífilis congênita no município de Belém (Pará): análise dos dados registrados nos sistemas de informação em saúde (SINAN, SIM e SINASC). 53 f. Dissertação (Saúde Pública) - Escola Nacional de Saúde Pública Sergio Arouca, Rio de Janeiro.

Holanda, M. T. C. G., Barreto, M. A., Machado, K. M. M. \& Pereira, R. C. (2011). Perfil epidemiológico da sífilis congênita no Município do Natal, Rio Grande do Norte - 2004 a 2007. Epidemiol. Serv. Saúde. 20(2).

Lafetá, K. R. G., Martelli Júnior. H., Silveira, M. F. \& Paranaíba, L. M. R. (2016). Sífilis materna e congênita, subnotificação e difícil controle. Revista Brasileira de Epidemiologia: 19, 63-74.

Lima, M. G., Santos, R. F. R., Barbosa, G. J. A. \& Ribeiro, G. S. (2013). Incidência e fatores de risco para sífilis congênita em Belo Horizonte, Minas Gerais, 2001-2008. Ciênc. saúde coletiva. 18(2), 499-506.

Magalhães, D. M. S., Kawaguchi, I. A. L., Dias, A. \& Calderon, I. M. P. (2013). Sífilis materna e congênita: ainda um desafio. Cad. Saúde Pública, 11091120 .

Melo, E. M. F. S. (2019). Sífilis congênita no Brasil: cenário de 2006 à 2018. Trabalho de Conclusão de Curso (Graduação em Enfermagem) - Faculdade de Ciências da Educação e Saúde, Centro Universitário de Brasília, Brasília.

Moreira, K. F. A., Oiveira, D. M., Alencar, L. N., Cavalcante, D. F. B., Pinheiro, A. S. \& Orfão, N. H. (2017). Perfil dos casos notificados de sífilis congênita. Revista Cogitare Enfermagem. Rondônia. 22(2).

Nunes, P. S., Zara, A. L. S. A., Rocha, D. F. N. C., Marinho, T. A., Mandacarú, P. M. P. \& Turchi, M. D. (2018). Sífilis gestacional e congênita e sua relação com a cobertura da Estratégia Saúde da Família, Goiás, 2007-2014: um estudo ecológico. Epidemiologia e Serviços de Saúde. 27(4).

Oliveira, D. R. \& Figueiredo, M. S. (2011). N. Abordagem conceitual sobre a sífilis na gestação e o tratamento de parceiros sexuais. Revista Enfermagem em Foco. Brasília. 2(2), 108-111.

Padovani, C., Oliveira, R. R. \& Pelloso, S. M. (2018). Syphilis in during pregnancy: association of maternal and perinatal characteristics in a region of southern Brazil. Revista Latino-Americana Enfermagem, Ribeirão Preto. 26.

Pereira, A. S., Shitsuka, D. M., Parreira, F. J. \& Shitsuka, R. (2018). Mtodologia da pesquisa científica. UFSM.

Reis, L. S. N., Freire, F. F. H., Gardoni, L. D., Guzella, M. V. V. M., Nobre, C. L., Laignier, G. V. \& Mansur, S. F. (2018). Perfil epidemiológico de sífilis congênita notificados no estado de Minas Gerais. Revista de Ciências, 9(25).

Rodrigues, V. L. R., Oliveira, F. M. \& Afonso, T. M. (2017). Sífilis congênita na perspectiva de um desafio para a saúde pública. Congresso internacional de enfermagem. Aracajú, SE. Anais.

Saraceni, V., Pereira, G. F. M., Silveira, M. F., Araujo, M. A. L. \& Miranda, A. E. (2017). Vigilância epidemiológica da transmissão vertical da sífilis: dados de seis unidades federativas no Brasil. Rev. Panam. Salud Publica, 41(44).

Teixeira, L. O., Belarmino, V., Gonçalves, C. V. \& Mendoza-Sassi, R. A. (2018). Tendência temporal e distribuição espacial da sífilis congênita no estado do Rio Grande do Sul entre 2001 e 2012. Revista Ciência e Saúde Coletiva. 23(8), 2587. 